\title{
A Nonlinear Predictive Controller Based on Chaos Optimization Apply to Reheated Steam Temperature
}

\author{
WU Wei-hong \\ Shanxi Electric Power Company Electric Power \\ Science Research Institute \\ Taiyuan, China
}

\author{
YAN Wei-ping, GUAN Zhi-min \\ North China Electric Power University \\ Baoding, China \\ xiaoguan79@sohu.com
}

\begin{abstract}
In the power plant reheat steam temperature control system with large time delay, large inertia and dynamic variation of uncertainty, a new nonlinear predictive controller is proposed which combines neural network identification, chaos optimization algorithm (COA) and the concept of predictive contro1. The controller utilizes neural network as predictive model and COA as online optimization. It can avoid calculating the complicated gradient and the inverse matrix in the nonlinear predictive control. The simulation studies show the effective performance of the proposed controller.
\end{abstract}

Keywords- reheated steam temperature; chaos optimization;nonlinear predictive control;neural network

\section{INTRODUCTION}

For large capacity, high parameter unit, in order to improve the circulation efficiency of steam turbine unit, prevent the final-stage water, requires the reheat system. Reheat steam temperature system is a typical nonlinear, time-varying, multivariable system, its state parameters and operating conditions are closely related ${ }^{11}$. Production process often have many interference factors, load, flue gas flow and temperature, combustion conditions within the furnace, air condition, water flow, pressure and temperature of heating surface fouling and pulverizing system such as start and stop of the reheat steam temperature has a great influence. There is an urgent need to design a robust control system, and can quickly into automatic control.

In recent years, due to the neural network played a significant role in the identification and control of nonlinear systems ${ }^{[2]}$, making it becomes a new tool for nonlinear model predictive control. Chaos optimization algorithm (COA) is a novel optimization method, it chaotic unique periodicity to achieve the global optimum, but it does not require the objective function of a continuous and differentiable nature.

In this paper, the neural network identification, chaos optimization and predictive control ideas organic combination of a new type of nonlinear model predictive controllers. The controller takes the neural network as a predictive model, the chaos optimization algorithm as a rolling optimization strategy to avoid the nonlinear model predictive control of complex gradient calculations and matrix inversion.

\section{THE DYNAMIC CHARACTERISTICS OF THE CFB BOILER BED TEMPERATURE}

The bed temperature is the control parameter related to the CFB boiler whether safe, efficient operation. This and the vapor pressure is a pair of strong coupling of variables, both by regulating the amount of fuel, primary air flow to achieve the control purposes. Literature [1], the proposed anti-system of the air-bed temperature to achieve complete decoupling control of the steam pressure and bed temperature Furthermore, the frequent movement of the air supply system is not conducive to the stability of the entire system, this paper considered by adjusting the amount of fuel to control the bed temperature.

CFB boiler in dynamic characteristics differ from those of pulverized coal furnace and oil furnace, which is mainly shown in CFB boiler combustion chamber fluidized layer of large capacity thermal balance characteristics and the operating conditions vary depending on the characteristic, caused the CFB boiler combustion process in automatic control difficult. When the load is large, the delay control object is correspondingly larger, it takes a long time to reach steady state, and the control difficulty is also a corresponding increase in.

\section{PRINCIPLE OF NONLINEAR PREDICTIVE CONTROL}

Considering the nonlinear system can be described as a discrete time model

$y(t)=f(y(t-1), \cdots, y(t-n), u(t-d), \cdots, u(t-d-m))(1)$

Among them: $n$ and $m$ respectively $y(t)$ and the output $u(t)$ of the order, $d$ is a nonlinear system of delay, $f(\cdot)$ is the unknown nonlinear functions. By the formula (1) expression of the object in time of $t+1$ the output predictive value

$\hat{y}(t+1)=f(y(t), \cdots, y(t-n+1), u(t-d+1), \cdots, u(t-d-m+1))(2)$

Use of equation (2) recursive available step $d$ ahead forecast

$\widehat{y}(t+d)=f(y(t+d-1), \cdots, y(t+d-n), u(t), \cdots, u(t-m))$

Used in equation (3) $t$ time and $t$ time earlier projections, replaced with actual output value 


$$
\widehat{y}(t+i-j)=\hat{y}(t+i-j)
$$

$\forall i-j \geq 0, j=1, \cdots, n$

A prediction horizon $N$, there are

$$
\begin{aligned}
& \hat{y}(t+i)=f(\hat{y}(t+i-1), \cdots, \hat{y}(t+i-n), u(t+i-d), \\
& \cdots, u(t-d-m+i))
\end{aligned}
$$

Among $i=1, \cdots N$

Quadratic objective function

$J=\sum_{j=N_{1}}^{N_{2}}\left[\hat{y}(t+j)-y_{r}(t+j)\right]^{2}+\lambda \sum_{j=N_{1}}^{N_{2}}[\Delta u(t+j-1)]^{2}$

Which: $N_{1}$ is the smallest prediction horizon (generally $N_{1}=d, d$ is system delay), $N_{2}$ is the largest of the prediction field, $N_{u}$ is to control the time, $\lambda>0$ is to control the weighting factor, $y_{r}(t+j)$ is for the moment $t+j$ of the reference trajectory value, set by the system value by the first-order filter softens after getting. Predictive control is aimed at controlling the amount of allowable interval for a group of optimal control incremental sequence $\left\{\Delta u *(t+j-1), j=1, \cdots, N_{u}\right\}$, makes the objective function $J$ minimum, in each sampling period only $\Delta u^{*}(t)$ to investment control, namely $u(t)=u(t-1)+\Delta u *(t)$, the optimization process of rolling ${ }^{[3]}$.

Based on the neural network for forecasting model, neural network output value as above predicted output values, rolling optimization strategy using chaos optimization algorithm (COA), the nonlinear model predictive controller structure shown in Figure 1. In the figure $a=1,2, \cdots, n, b=d, d+1, \cdots, d+m$.

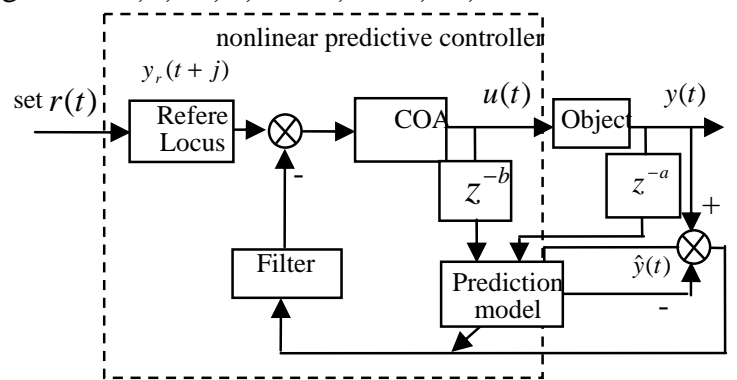

Figure 1. Structure of nonlinear predictive controller

\section{ROLLING OPTIMIZATION BASED ON COA}

Chaos optimization using chaotic variables to the solution space searching, its basic idea is: first make the optimization variables in phase space is in a chaotic state ${ }^{[4]}$, which produced a group with the same number of optimization variables of chaotic variables, using a similar carrier way putting chaos into the optimization variables make its chaotic, and the chaotic motion traversing range amplified to optimize the scope of variables, and then directly using chaos search.

Rolling optimization in nonlinear predictive control strategy is a nonlinear programming problem. Compared to conventional linear programming method with the simplex method, quadratic programming, chaos optimization algorithm due to ergodicity, randomness, and so can more efficiently solve the optimization problem is easy to implement. Based on this consideration, this article will be chaos optimization algorithm used to rolling optimization in nonlinear predictive control, which is limited within the control sequence optimization.

This paper uses the Logistic mapping

$$
x_{n+1}=\mu x_{n}\left(1-x_{n}\right), x_{0} \in(0,1)
$$

Which: $\mu$ is the control parameter, when $\mu=4$, Logistic mapping on the $(0,1)$ interval mapping is full, and the system is in a chaotic state, $x_{n}$ is in the context of $(0,1)$ traverse. Due to the chaotic state of the initial values are extremely sensitive characteristics, in the range $(0,1)$ of (6) take a number of slightly different initial value, can get several different trajectories of the chaotic variables $\left\{\Delta u *(t+j-1), j=1, \cdots, N_{u}\right\}$, which attract the sub-field mapping symmetric about the origin. To be selected for the $N_{u}$ chaotic variables are a carrier and iteration, two carrier and iteration, finally get the current optimal solution.

The basic steps of chaos optimization algorithm for solving optimal control are as follows:

1) Initialization algorithm ${ }^{[5]}$ : randomly taken from the $N_{u}$ initial value with small differences in the interval $(0,1)$, respectively, substituted into equation (6), can be $N_{u}$ chaotic trajectory variables $x_{j}\left(j=1, \cdots, N_{u}\right)$

2) A carrier: by equation (6) with a carrier method will be $N_{u}$ chaotic variables $x_{j}\left(j=1, \cdots, N_{u}\right)$ are introduced into the objective function (5) of the $N_{u}$ optimizing parameters, and the chaotic variables $x_{j}\left(j=1, \cdots, N_{u}\right)$ are adjusted to the scope of optimize variation $\Delta u(t+j-1)$ :

$\Delta u^{*}(t+j-1)=p_{j}+q_{j} \cdot x_{j}, j=1,2, \cdots, N_{u}$

Where: $\quad p_{j} \quad$ and $\quad q_{j}$ are constants, $p_{j}=\Delta u_{\min }, q_{j}=\Delta u_{\max }-\Delta u_{\min }$

3) Iterative search using chaotic variables.

Calculate the $\Delta u^{\prime}(t+j-1), j=1,2, \cdots, N_{u}$ corresponding performance indicators $J^{\prime}$, if $J^{\prime} \leq J^{*}\left(J^{*}\right.$ is the index value for the current optimal solution $\Delta u^{*}(t+j-1)$ corresponding

to), 
$J^{*}=J^{\prime}, \Delta u^{*}(t+j-1)=\Delta u^{\prime}(t+j-1), j=1,2, \cdots, N_{u}$, otherwise remain unchanged.

4) Secondary carrier: remain the same $J^{*}$ if after step 3) several-step search, according to equation (7) to the secondary carrier:

$$
\Delta u^{\prime \prime}(t+j-1)=\Delta u^{*}(t+j-1)+\beta \Delta u^{*}(t+j-1)\left(0.5-x_{j}\right)
$$

$, j=1,2, \cdots, N_{u}$

Among them: $\beta$ is an adjustable parameter, can be less than 1 ; conversely, returned to step 3 ).

5) Chaos variables in the secondary carrier to continue the iterative search ${ }^{[6]}$.

$$
\text { Calculation }
$$

the

$\Delta u^{\prime \prime}(t+j-1) \quad, \quad j=1,2, \cdots, N_{u} \quad$ corresponding performance indicators $J^{\prime \prime}$, if $J^{\prime \prime} \leq J^{*} \quad$,then $\quad J^{*}=J^{\prime \prime}$, $\Delta u^{*}(t+j-1)=\Delta u^{\prime \prime}(t+j-1) \quad, \quad j=1,2, \cdots, N_{u}$, otherwise remain unchanged.

6) If the termination condition is satisfied, is to terminate search, otherwise return to step 5).

\section{SimULATION STUDY}

The chaotic neural network model for predicting optimal control decision is applied to the nonlinear, large delay boiler reheat steam temperature control. Figure 2 is the forward - feedback cascade control system block diagram.

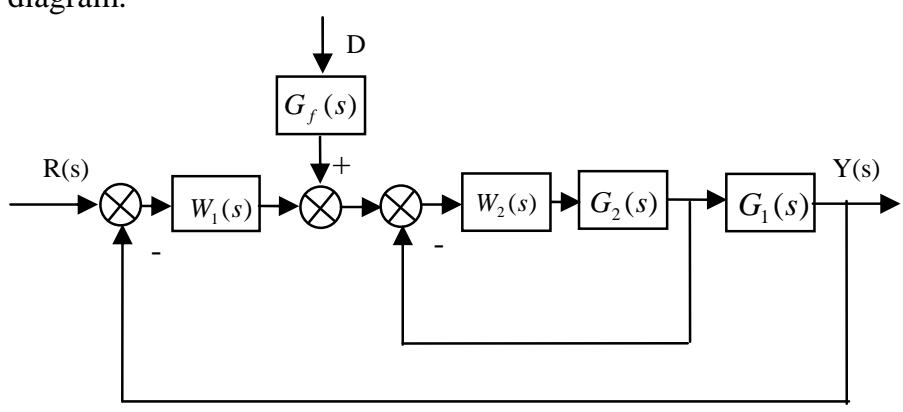

Figure 2. The forward - feedback cascade control system block diagram

Here, the main controller $W_{1}(s)$ is the optimal control of the chaotic neural network prediction model, deputy controller $W_{2}(s)$ with proportional control, the scale factor setting is 5 .

\section{A.Step test}

The nonlinear predictive controller based on chaos optimization is applied to the reheated steam temperature control system, the unit step signal $r(t)$ for input signal, and the system $75 \%$ load response tests. The step response curve of the reheated steam temperature shown in Figure 3, in figure, $\mathrm{y}$ is the output amplitude of dimension 1.

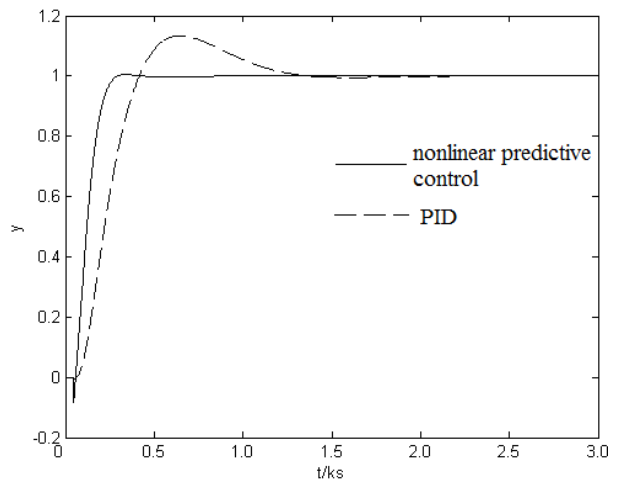

Figure 3. the step response curve of $75 \%$ load

Compared with PID control reheated steam temperature system, the use of nonlinear model predictive control, the overshoot is less than conventional PID control, the entire transition process even smoother, shorter time of process control.

\section{B.Disturbance test}

With $75 \%$ load condition as an example, suppose the object regulated by square wave disturbance $(\mathrm{n}(\mathrm{t}))$, as shown in figure 4 . The simulation time is $5 \mathrm{ks}$, in $3 \mathrm{ks}$ the amplitude of the square wave disturbance is 0.1 , disturbance time is 3000s 3500s.

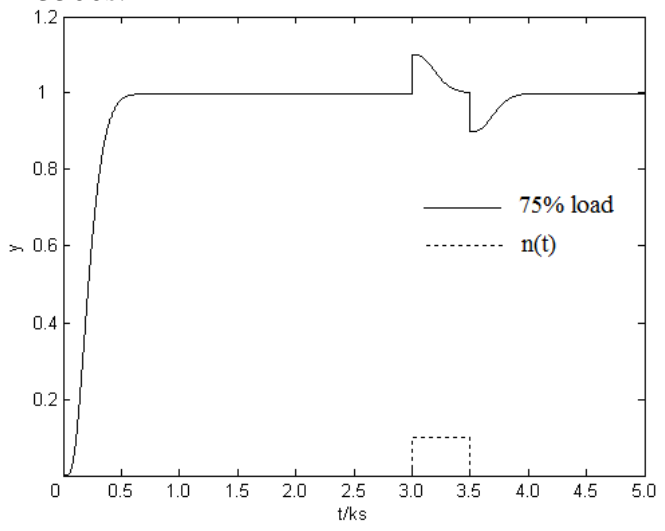

Figure 4. Step response curve by disturbance $n(t)$

A large number of simulation experiments show that, the nonlinear predictive controller has good real-time performance and anti-jamming ability for uncertain large delay systems.

\section{CONCLUSIONS}

Chaos optimization algorithm is a new global optimization method. It can effectively deal with the optimization problems of nonlinear system predictive control, avoiding the complex gradient calculation and matrix inversion.

Based on chaos optimization theory and its application in nonlinear predictive control are studied, and combined with 
the actual thermal process (reheated steam temperature) predictive control is simulated, simulation results show that chaotic optimization is effective, the nonlinear predictive controller has good real time and tracking performance, achieved good control effect.

\section{REFERENCES}

[1] WANG Wenbiao, LI Jinping, YOU Wenming. Adjustment on reheating steam temperature of 300 MW thermal power plant [J]. Shan Xi Electric Power, 2001, 6: 26-28.

[2] ZHANG Xinghui,DU Shengzhi,CHEN Zengqiang,et a1 . A constrained nonlinear predictive control based on genetic
algorithm[J]. Chinese J of Scientific Instrument,2003,24(S4) : 556558

[3] ZHU Xuefeng, Dale E. Seborg. Nonlinear Predictive Control Based on Hammerstein Models [J]. Control Theory and Applications, 1994, 11(5): 564-575

[4] DOU Chunxia. Fuzzy Forecast Controller Based on Chaos Neural Network Model Research and Its Application [J]. Journal of System Simulation, 2002, 14(10): 1372-1375

[5] LI Bing,JIANG Weisun . Chaos optimization method and its application[J] . Control Theory \& Applications,1997,14(4) : 613615.

[6] LI Xiang,CHEN Zengqiang,YUAN Zhuzhi . Study on chaos mechanism in system identification using T-S model fuzzy neural networks[J] . Control and Decision,2001,16(4) : 504-506 . 